\title{
FORCED VIBRATIONS OF A THERMOELASTIC DOUBLE POROUS MICROBEAM SUBJECTED TO A MOVING LOAD
}

\author{
RAJNEESH KuMAR \\ Kurukshetra University, Department of Mathematics, Kurukshetra, Haryana, India \\ RICHA VOHRA \\ H.P. University, Department of Mathematics and Statistics, Shimla, HP, India \\ e-mail: richavhr88@gmail.com
}

\begin{abstract}
The present paper deals with forced vibrations of a homogeneous, isotropic thermoelastic double porous microbeam subjected to moving load, in context of Lord-Shulman theory of thermoelasticity with one relaxation time. The Laplace transform has been applied to obtain expressions for the axial displacement, lateral deflection, volume fraction field and temperature distribution. A numerical inversion technique has been used to recover the resulting quantities in the physical domain. Effects of velocity and time parameters are shown graphically by plotting axial displacement, lateral deflection, volume fraction field and temperature distribution against distance. Some particular cases are also deduced.
\end{abstract}

Keywords: double porosity, thermoelasticity, Lord-Shulman theory, microbeam, moving load

\section{Introduction}

Recently, dynamical analysis of engineering structures subjected to moving loads has gained great importance. Vehicle-bridge interactions are a vast area of interest in the moving load problem. Advances in transport technology and automobile engineering have resulted in high speeds and heaviness of vehicles and other moving bodies. As a result, corresponding structures have been subjected to vibration and dynamic stress much higher than ever before. The engineering structures with moving loads often come out in buildings, bridges, railways and cranes. Beam type structures are widely used in many fields like civil, mechanical and aerospace engineering. Many researchers have investigated dynamical behavior of beams on elastic foundations subjected to moving loads, especially in railway engineering. The modern trend towards higher speeds in the railways has further intensified the research in order to accurately predict the vibration behavior of railway tracks.

Pores or fractures can be observed in engineering structures due to reasons like erosion, corrosion, fatigue or accidents which affect the dynamic behavior of the entire structure to a considerable extent. This leads to the development of the double porosity model which has its applications in geophysics, rock mechanics and many branches of engineering like civil engineering, chemical engineering and the petroleum industry. Biot (1941) proposed a model for porous media with single porosity. Later on Barenblatt et al. (1960) introduced a model for porous media with a double porosity structure. The double porosity model consists of two coexisting degrees of porosity in which one corresponds to the porous matrix and the other to the fissure matrix.

Nunziato and Cowin (1979) developed a nonlinear theory of an elastic material with voids. Later, Cowin and Nunziato (1983) developed a theory of linear elastic materials with voids for mathematical study of the mechanical behavior of porous solids. In this theory, the skeletal materials are elastic, and interstices are void of material, hence an additional degree of freedom, 
the volume fraction of the void, is added. Iesan and Quintanilla (2014) derived a theory of thermoelastic solids with a double porosity structure by using the theory developed by Nunziato and Cowin (1979). Darcy's law was not used in developing that theory. So far, not much work has been done on the theory of thermoelasticity with the double porosity based on the model proposed by Iesan and Quintanilla (2014). Recent investigations have been started in the theory of thermoelasticity with double porosity which has a significant application in continuum mechanics. Kumar et al. (2015) applied the state space approach to a boundary value problem for thermoelastic materials with double porosity.

The dynamic behavior of different isotropic structures subjected to moving loads has been investigated by many researchers. Olsson (1991) studied the dynamic problem of a simply supported beam subjected to a constant force moving at a constant speed. The linear dynamic response of a simply supported uniform beam under a moving load of constant magnitude and velocity was investigated by Michaltsos et al. (1996). Rao (2000) studied the dynamic response of a multi-span Euler-Bernoulli beam due to moving loads. Mehri et al. (2009) presented the linear dynamic response of uniform beams with different boundary conditions under a moving load based on the Euler-Bernoulli beam theory. Sharma and Grover (2011) analysed a thermoelastic vibrations in micro-/nano-scale beam resonators with the presence of voids. Kargarnovin et al. (2012) studied the dynamic response of a delaminated composite beam under the action of a moving oscillatory mass. Esen (2015) investigated the transverse and longitudinal vibrations of a thin plate which carried a load moving along an arbitrary trajectory with variable velocity. Kumar (2016) studied the response of a thermoelastic beam due to the thermal source in the modified couple stress theory. Kaghazian et al. (2017) investigated free vibrations of a piezoelectric nanobeam using nonlocal elasticity theory. Zenkour (2017) studied the thermoelastic response of a microbeam embedded in visco-Pasternak's medium based on GN-III model.

In the present work, forced vibrations of a homogeneous, isotropic thermoelastic double porous microbeam, subjected to a moving load in the context of Lord-Shulman theory of thermoelasticity has been investigated. The Laplace transform has been applied to find expressions for axial displacement, lateral deflection, volume fraction fields and temperature distribution. The resulting quantities are obtained in the physical domain by using a numerical inversion technique. Variations of the axial displacement, lateral deflection, volume fraction field and temperature distribution against the axial distance are depicted graphically to show the effect of the velocity parameter. Some particular cases have also been deduced.

\section{Basic equations}

Following Iesan and Quintanilla (2014) as well as Lord and Shulman (1967); the field equations and the constitutive relation for a homogeneous isotropic thermoelastic material with a double porosity structure in the absence of body forces, extrinsic equilibrated body forces and heat sources can be written as

$$
\begin{aligned}
& \mu \nabla^{2} u_{i}+(\lambda+\mu) u_{j, j i}+b \varphi_{, i}+d \psi_{, i}-\beta T_{, i}=\rho \ddot{u}_{i} \\
& \alpha \nabla^{2} \varphi+b_{1} \nabla^{2} \psi-b u_{r, r}-\alpha_{1} \varphi-\alpha_{3} \psi+\gamma_{1} T=\kappa_{1} \ddot{\varphi} \\
& b_{1} \nabla^{2} \varphi+\gamma \nabla^{2} \psi-d u_{r, r}-\alpha_{3} \varphi-\alpha_{2} \psi+\gamma_{2} T=\kappa_{2} \ddot{\psi}
\end{aligned}
$$

and

$$
\begin{aligned}
& \left(1+\tau_{0} \frac{\partial}{\partial t}\right)\left(\beta T_{0} \dot{u}_{j, j}+\gamma_{1} T_{0} \dot{\varphi}+\gamma_{2} T_{0} \dot{\psi}+\rho C^{*} \dot{T}\right)=K^{*} \nabla^{2} T \\
& t_{i j}=\lambda e_{r r} \delta_{i j}+2 \mu e_{i j}+b \varphi \delta_{i j}+d \psi \delta_{i j}-\beta T \delta_{i j}
\end{aligned}
$$


where $\lambda$ and $\mu$ are Lame's constants, $\rho$ is mass density, $\beta=(3 \lambda+2 \mu) \alpha_{t}, \alpha_{t}$ is linear thermal expansion, $C^{*}$ is specific heat at a constant strain, $u_{i}$ are displacement components, $t_{i j}$ is the stress tensor, $\kappa_{1}$ and $\kappa_{2}$ are coefficients of equilibrated inertia, $\varphi$ is the volume fraction field corresponding to pores, and $\psi$ is the volume fraction field corresponding to fissures, $K^{*}$ is the coefficient of thermal conductivity, $\tau_{0}$ is the thermal relaxation time, $\kappa_{1}$ and $\kappa_{2}$ are coefficients of equilibrated inertia, and $b, d, b_{1}, \gamma, \gamma_{1}, \gamma_{2}$ are constitutive coefficients, $\delta_{i j}$ is Kronecker's delta, $T$ is the temperature change measured form the absolute temperature $T_{0}\left(T_{0} \neq 0\right)$, a superposed dot represents differentiation with respect to time variable $t$.

\section{Formulation of the problem}

We consider a homogeneous, isotropic thermoelastic double porous microbeam having dimensions: length $L(0 \leqslant x \leqslant L)$, width $a(-a / 2 \leqslant y \leqslant a / 2)$ and thickness $h(-h / 2 \leqslant z \leqslant h / 2)$ in a Cartesian coordinate sytem $O x y z$ as shown in Fig. 1. The microbeam undergoes bending vibrations of a small amplitude about the $x$-axis such that the deflection is consistent with the linear Euler-Bernoulli theory. Therefore, the displacements can be written as

$$
u_{1}=u=-z \frac{\partial w}{\partial x} \quad u_{2}=0 \quad u_{3}=w(x, t)
$$

where $w$ is the lateral deflection and $u$ is the axial displacement.

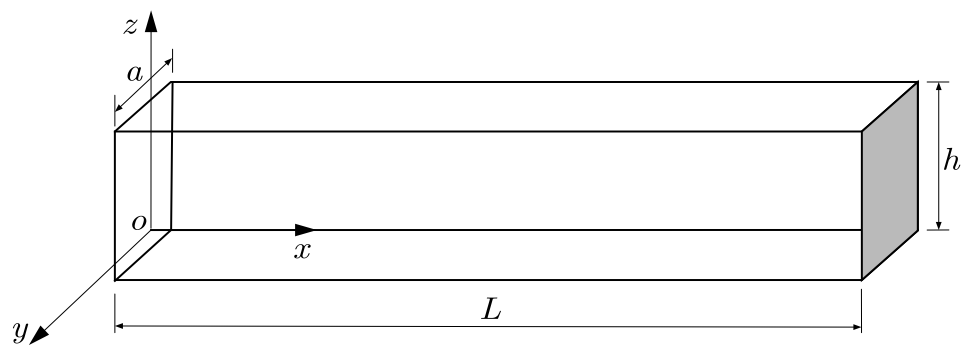

Fig. 1. Geometry of the beam

The equation of motion for forced vibrations of the beam can be written as

$$
\frac{\partial^{2} M}{\partial x^{2}}+\rho A \frac{\partial^{2} w}{\partial t^{2}}=F(x, t)
$$

where $A=a h$ is the cross-section area, $M$ is the flexural moment of cross section of the microbeam and $F(x, t)$ is the applied moving load. By substituting Eqs. (2.3) and (3.1) into Eq.(3.2), we obtain the equation of motion for forced vibrations of an Euler-Bernoulli thermoelastic double porous microbeam subjected to moving load as

$$
(\lambda+2 \mu) I \frac{\partial^{4} w}{\partial x^{4}}+\rho A \frac{\partial^{2} w}{\partial t^{2}}-\frac{\partial^{2} M_{\varphi}}{\partial x^{2}}-\frac{\partial^{2} M_{\psi}}{\partial x^{2}}+\frac{\partial^{2} M_{T}}{\partial x^{2}}=F(x, t)
$$

where $F=F_{0} \delta(x-v t)$ is the applied moving load, $v$ is its velocity, $\delta$ is the Dirac delta function, $t$ is time in seconds, $I=a h^{3} / 12$ is the moment of inertia of the cross-section and $M_{\varphi}, M_{\psi}$ are the volume fraction field moments, and $M_{T}$ is the thermal moment of the beam given by

$$
M_{\varphi}=b \int_{-h / 2}^{h / 2} a \varphi z d z \quad M_{\psi}=d \int_{-h / 2}^{h / 2} a \psi z d z \quad M_{T}=\beta \int_{-h / 2}^{h / 2} a T z d z
$$


Equations (2.1) 2,3 and (2.2) with the help of Eq. (3.1) can be written as

$$
\begin{aligned}
& \alpha\left(\frac{\partial^{2} \varphi}{\partial x^{2}}+\frac{\partial^{2} \varphi}{\partial z^{2}}\right)+b_{1}\left(\frac{\partial^{2} \psi}{\partial x^{2}}+\frac{\partial^{2} \psi}{\partial z^{2}}\right)+b z \frac{\partial^{2} w}{\partial x^{2}}-\alpha_{1} \varphi-\alpha_{3} \psi+\gamma_{1} T=\kappa_{1} \frac{\partial^{2} \varphi}{\partial t^{2}} \\
& b_{1}\left(\frac{\partial^{2} \varphi}{\partial x^{2}}+\frac{\partial^{2} \varphi}{\partial z^{2}}\right)+\gamma\left(\frac{\partial^{2} \psi}{\partial x^{2}}+\frac{\partial^{2} \psi}{\partial z^{2}}\right)+d z \frac{\partial^{2} w}{\partial x^{2}}-\alpha_{3} \varphi-\alpha_{2} \psi+\gamma_{2} T=\kappa_{2} \frac{\partial^{2} \psi}{\partial t^{2}} \\
& K^{*}\left(\frac{\partial^{2} T}{\partial x^{2}}+\frac{\partial^{2} T}{\partial z^{2}}\right)=\left(1+\tau_{0} \frac{\partial}{\partial t}\right)\left[-\beta T_{0} z \frac{\partial}{\partial t}\left(\frac{\partial^{2} w}{\partial x^{2}}\right)+\gamma_{1} T_{0} \dot{\varphi}+\gamma_{2} T_{0} \dot{\psi}+\rho C^{*} \dot{T}\right]
\end{aligned}
$$

\section{Solution of the problem}

For the present microbeam, we assume that there is no flow of heat and the volume fraction fields across the surfaces $(z= \pm h / 2)$ so that $\partial T / \partial z=\partial \varphi / \partial z=\partial \psi / \partial z=0$ at $z= \pm h / 2$. For a very thin beam, assuming that the volume fraction fields and temperature increment in terms of $\sin (\pi z / h)$ function along the thickness direction one obtains

$$
\varphi(x, z, t)=\Phi(x, t) \sin \frac{\pi z}{h} \quad \psi(x, z, t)=\Psi(x, t) \sin \frac{\pi z}{h} \quad T(x, z, t)=\Theta(x, t) \sin \frac{\pi z}{h}
$$

Introducing non-dimensional variables as

$$
\begin{aligned}
& x^{\prime}=\frac{1}{L} x \quad u^{\prime}=\frac{1}{L} u \quad t_{x}^{\prime}=\frac{t_{x}}{E} \\
& \Phi^{\prime}=\frac{L}{\alpha} \Phi \quad \Psi^{\prime}=\frac{L}{\alpha} \Psi \quad \Theta^{\prime}=\frac{\beta}{E} \Theta \\
& t^{\prime}=\frac{c_{1}}{L} t \quad \tau_{0}^{\prime}=\frac{c_{1}}{L} \tau_{0} \quad F_{0}^{\prime}=\frac{L^{2}}{a h^{2}(\lambda+2 \mu)} F_{0}
\end{aligned}
$$

where $c_{1}^{2}=(\lambda+2 \mu) / \rho$ and $E=\mu(3 \lambda+2 \mu) /(\lambda+\mu)$ is Young's modulus.

Making use of Eqs. (4.1) in Eq. (3.3) and with the aid of Eqs. (4.2), yields (after suppressing primes)

$$
\frac{\partial^{4} w}{\partial x^{4}}+a_{1} \frac{\partial^{2} w}{\partial t^{2}}-a_{2} \frac{\partial^{2} \Phi}{\partial x^{2}}-a_{3} \frac{\partial^{2} \Psi}{\partial x^{2}}+a_{4} \frac{\partial^{2} \Theta}{\partial x^{2}}=F_{0} \delta(x-v t)
$$

On multiplying Eqs. (3.5) by $z$ and integrating them with respect to $z$ from $-h / 2$ to $h / 2$ and after using Eq.(4.2), we obtain (suppressing primes for convenience)

$$
\begin{aligned}
& a_{5} \frac{\partial^{2} \Phi}{\partial x^{2}}-a_{6} \Phi+a_{7} \frac{\partial^{2} \Psi}{\partial x^{2}}-a_{8} \Psi+a_{9} \frac{\partial^{2} w}{\partial x^{2}}-a_{10} \Phi-a_{11} \Psi+a_{12} \Theta=\frac{\partial^{2} \Phi}{\partial t^{2}} \\
& a_{13} \frac{\partial^{2} \Phi}{\partial x^{2}}-a_{14} \Phi+a_{15} \frac{\partial^{2} \Psi}{\partial x^{2}}-a_{16} \Psi+a_{17} \frac{\partial^{2} w}{\partial x^{2}}-a_{18} \Phi-a_{19} \Psi+a_{20} \Theta=\frac{\partial^{2} \Psi}{\partial t^{2}}
\end{aligned}
$$

and

$$
\frac{\partial^{2} \Theta}{\partial x^{2}}-a_{21} \Theta=\left(1+\tau_{0} \frac{\partial}{\partial t}\right)\left[a_{22} \frac{\partial}{\partial t}\left(\frac{\partial^{2} w}{\partial x^{2}}\right)+a_{23} \frac{\partial \Phi}{\partial t}+a_{24} \frac{\partial \Psi}{\partial t}+a_{25} \frac{\partial \Theta}{\partial t}\right]
$$


where

$$
\begin{array}{rlrl}
a_{1}=\frac{\rho c_{1}^{2} L}{I(\lambda+2 \mu)} & a_{2}=\frac{2 b \alpha}{I \pi^{2}(\lambda+2 \mu) L} & a_{3}=\frac{2 d \alpha}{I \pi^{2}(\lambda+2 \mu) L} \\
a_{4}=\frac{2 E}{I \pi^{2}(\lambda+2 \mu)} & a_{5}=\frac{\alpha}{\kappa_{1} c_{1}^{2}} & a_{6}=\frac{\alpha \pi^{2} L^{2}}{\kappa_{1} c_{1}^{2} h^{2}} & a_{7}=\frac{b_{1}}{\kappa_{1} c_{1}^{2}} \\
a_{8}=\frac{b_{1} \pi^{2} L^{2}}{\kappa_{1} c_{1}^{2} h^{2}} & a_{9}=\frac{b h \pi^{2} L^{2}}{24 \alpha \kappa_{1} c_{1}^{2}} & a_{10}=\frac{\alpha_{1} L^{2}}{\kappa_{1} c_{1}^{2}} & a_{11}=\frac{\alpha_{3} L^{2}}{\kappa_{1} c_{1}^{2}} \\
a_{12}=\frac{\gamma_{1} E L^{3}}{\alpha \beta \kappa_{1} c_{1}^{2}} & a_{13}=\frac{b_{1}}{\kappa_{2} c_{1}^{2}} & a_{14}=\frac{b_{1} \pi^{2} L^{2}}{\kappa_{2} c_{1}^{2} h^{2}} & a_{15}=\frac{\gamma}{\kappa_{2} c_{1}^{2}} \\
a_{16}=\frac{\gamma \pi^{2} L^{2}}{\kappa_{2} c_{1}^{2} h^{2}} & a_{17}=\frac{d h \pi^{2} L^{2}}{24 \alpha \kappa_{2} c_{1}^{2}} & a_{18}=\frac{\alpha_{3} L^{2}}{\kappa_{2} c_{1}^{2}} & a_{19}=\frac{\alpha_{2} L^{2}}{\kappa_{2} c_{1}^{2}} \\
a_{20}=\frac{\gamma_{2} E L^{3}}{\alpha \beta \kappa_{2} c_{1}^{2}} & a_{21}=\frac{\pi^{2} L^{2}}{h^{2}} & a_{22}=-\frac{\beta^{2} T_{0} h c_{1} \pi^{2}}{24 E K^{*}} & \\
a_{23}=\frac{\alpha \beta T_{0} \gamma_{1} c_{1}}{E K^{*}} & a_{24}=\frac{\alpha \beta T_{0} \gamma_{2} c_{1}}{E K^{*}} & a_{25}=\frac{\rho C^{*} c_{1} L}{K^{*}} &
\end{array}
$$

The initial conditions of the problem are assumed to be homogeneous and are taken as

$$
\begin{aligned}
& \left.w(x, t)\right|_{t=0}=\left.\frac{\partial w(x, t)}{\partial t}\right|_{t=0}=\left.\Phi(x, t)\right|_{t=0}=\left.\frac{\partial \Phi(x, t)}{\partial t}\right|_{t=0}=\left.\Psi(x, t)\right|_{t=0}=\left.\frac{\partial \Psi(x, t)}{\partial t}\right|_{t=0}=0 \\
& \left.\Theta(x, t)\right|_{t=0}=\left.\frac{\partial \Theta(x, t)}{\partial t}\right|_{t=0}=0
\end{aligned}
$$

These initial conditions are supplemented by considering that the two ends of the microbeam are clamped and remain at zero increment of the volume fraction fields and temperature. Mathematically, it can be written as

$$
\begin{aligned}
& \left.w(x, t)\right|_{x=0, L}=\left.\frac{\partial w(x, t)}{\partial x}\right|_{x=0, L}=\left.0 \quad \Phi(x, t)\right|_{x=0, L}=0 \\
& \left.\Psi(x, t)\right|_{x=0, L}=\left.0 \quad \Theta(x, t)\right|_{x=0, L}=0
\end{aligned}
$$

\section{Solution in the Laplace transform domain}

Applying the Laplace transform defined by

$$
\bar{f}(s)=L[f(t)]=\int_{0}^{\infty} f(t) \mathrm{e}^{-s t} d t
$$

to Eqs. (4.3)-(4.5) under initial conditions (4.6), and after some simplifications, we obtain

$$
\left(\frac{d^{10}}{d x^{10}}+B_{1} \frac{d^{8}}{d x^{8}}+B_{2} \frac{d^{6}}{d x^{6}}+B_{3} \frac{d^{4}}{d x^{4}}+B_{4} \frac{d^{2}}{d x^{2}}+B_{5}\right)(\bar{w}, \bar{\Phi}, \bar{\Psi}, \bar{\Theta})=\left(f_{1}, f_{2}, f_{3}, f_{4}\right) \mathrm{e}^{-\frac{s}{v} x}
$$

where $B_{1}, B_{2}, B_{3}, B_{4}, B_{5}, f_{1}, f_{2}, f_{3}, f_{4}$, are given in Appendix I. 
The solution to system of Eqs. (5.2), in the Laplace transform domain, can be written as

$$
\begin{aligned}
& \bar{w}=H_{1} \mathrm{e}^{-\frac{s}{v} x}+\sum_{i=1}^{5}\left(D_{i} \mathrm{e}^{-m_{i} x}+D_{i+5} \mathrm{e}^{m_{i} x}\right) \\
& \bar{\Phi}=H_{2} \mathrm{e}^{-\frac{s}{v} x}+\sum_{i=1}^{5} g_{1 i}\left(D_{i} \mathrm{e}^{-m_{i} x}+D_{i+5} \mathrm{e}^{m_{i} x}\right) \\
& \bar{\Psi}=H_{3} \mathrm{e}^{-\frac{s}{v} x}+\sum_{i=1}^{5} g_{2 i}\left(D_{i} \mathrm{e}^{-m_{i} x}+D_{i+5} \mathrm{e}^{m_{i} x}\right) \\
& \bar{\Theta}=H_{4} \mathrm{e}^{-\frac{s}{v} x}+\sum_{i=1}^{5} g_{3 i}\left(D_{i} \mathrm{e}^{-m_{i} x}+D_{i+5} \mathrm{e}^{m_{i} x}\right)
\end{aligned}
$$

where

$$
H_{i}=\frac{f_{i} \nu^{10}}{s^{10}+B_{1} s^{8} \nu^{2}+B_{2} s^{6} \nu^{4}+B_{3} s^{4} \nu^{6}+B_{4} s^{2} \nu^{8}+B_{5} \nu^{10}} \quad i=1,2,3,4
$$

and $g_{1 i}, g_{2 i}, g_{3 i} ;(i=1,2,3,4,5)$ are given in Appendix II.

Here $\pm m_{i}, i=1,2, \ldots, 5$ are the roots of the characteristic equation

$$
m^{10}+B_{1} m^{8}+B_{2} m^{6}+B_{3} m^{4}+B_{4} m^{2}+B_{5}=0
$$

Therefore, the corresponding expressions for the axial displacement in the Laplace transform domain can be written as

$$
\bar{u}=-z \frac{d \bar{w}}{d x}=-z\left[\sum_{i=1}^{5}\left(-m_{i} D_{i} \mathrm{e}^{-m_{i} x}+m_{i} D_{i+5} \mathrm{e}^{m_{i} x}\right)-\frac{s}{v} H_{1} \mathrm{e}^{-\frac{s}{v} x}\right]
$$

Boundary conditions (4.7) in the Laplace transform domain take the form as

$$
\begin{aligned}
& \left.\bar{w}(x, s)\right|_{x=0, L}=\left.\frac{d \bar{w}(x, s)}{\partial x}\right|_{x=0, L}=\left.0 \quad \bar{\Phi}(x, s)\right|_{x=0, L}=0 \\
& \left.\bar{\Psi}(x, s)\right|_{x=0, L}=\left.0 \quad \bar{\Theta}(x, s)\right|_{x=0, L}=0
\end{aligned}
$$

By substituting Eqs. (5.3) into boundary conditions (5.5), we obtain a system of ten linear equations in the matrix form as

$$
\mathrm{AD}=\mathbf{E}
$$

where

$$
\begin{aligned}
& \mathbf{A}=\left[\begin{array}{cccccccccc}
1 & 1 & 1 & 1 & 1 & 1 & 1 & 1 & 1 & 1 \\
b_{1} & b_{2} & b_{3} & b_{4} & b_{5} & a_{1} & a_{2} & a_{3} & a_{4} & a_{5} \\
-m_{1} & -m_{2} & -m_{3} & -m_{4} & -m_{5} & m_{1} & m_{2} & m_{3} & m_{4} & m_{5} \\
-m_{1} b_{1} & -m_{2} b_{2} & -\lambda_{3} b_{3} & -m_{4} b_{4} & -m_{5} b_{5} & m_{1} a_{1} & m_{2} a_{2} & m_{3} a_{3} & m_{4} a_{4} & m_{5} a_{5} \\
g_{11} & g_{12} & g_{13} & g_{14} & g_{15} & g_{11} & g_{12} & g_{13} & g_{14} & g_{15} \\
g_{11} b_{1} & g_{12} b_{2} & g_{13} b_{3} & g_{14} b_{4} & g_{15} b_{5} & g_{11} a_{1} & g_{12} a_{2} & g_{13} a_{3} & g_{14} a_{4} & g_{15} a_{5} \\
g_{21} & g_{22} & g_{23} & g_{24} & g_{25} & g_{21} & g_{22} & g_{23} & g_{24} & g_{25} \\
g_{21} b_{1} & g_{22} b_{2} & g_{23} b_{3} & g_{24} b_{4} & g_{25} b_{5} & g_{21} a_{1} & g_{22} a_{2} & g_{23} a_{3} & g_{24} a_{4} & g_{25} a_{5} \\
g_{31} & g_{32} & g_{33} & g_{34} & g_{35} & g_{31} & g_{32} & g_{33} & g_{34} & g_{35} \\
g_{31} b_{1} & g_{32} b_{2} & g_{33} b_{3} & g_{34} b_{4} & g_{35} b_{5} & g_{31} a_{1} & g_{32} a_{2} & g_{33} a_{3} & g_{34} a_{4} & g_{35} a_{5}
\end{array}\right] \\
& a_{i}=\mathrm{e}^{m_{i} L} \quad b_{i}=\mathrm{e}^{-m_{i} L} \quad i=1,2,3,4,5 \\
& \mathbf{D}=\left[\begin{array}{llllllllll}
D_{1} & D_{2} & D_{3} & D_{4} & D_{5} & D_{6} & D_{7} & D_{8} & D_{9} & D_{10}
\end{array}\right]^{\mathrm{T}} \\
& \mathbf{E}=\left[\begin{array}{llllllllll}
E_{1} & E_{2} & E_{3} & E_{4} & E_{5} & E_{6} & E_{7} & E_{8} & E_{9} & E_{10}
\end{array}\right]^{\mathrm{T}}
\end{aligned}
$$


and

$$
\begin{array}{llll}
E_{1}=-H_{1} & E_{2}=-H_{1} \mathrm{e}^{-\frac{s}{v} L} & E_{3}=\frac{s}{v} H_{1} & E_{4}=\frac{s}{v} H_{1} \mathrm{e}^{-\frac{s}{v} L} \\
E_{5}=-H_{2} & E_{6}=-H_{2} \mathrm{e}^{-\frac{s}{v} L} & E_{7}=-H_{3} & E_{8}=-H_{3} \mathrm{e}^{-\frac{s}{v} L} \\
E_{9}=-H_{4} & E_{10}=-H_{4} \mathrm{e}^{-\frac{s}{v} L} & &
\end{array}
$$

By solving the above system of equations (5.6), we obtain the values of unknown parameters $D_{i}$, $i=1,2, \ldots, 10$.

This completes the solution of the problem in the Laplace transform domain.

In order to determine the axial displacement, lateral deflection, volume fraction field and temperature distribution in the physical domain, we will adopt a numerical inversion method given by Honig and Hirdes (1984).

In this method, the Laplace domain $\bar{f}(s)$ can be inverted to the time domain $f(t)$ as

$$
f(t)=\frac{1}{t_{1}} \exp (\Omega t)\left[\frac{1}{2} \bar{f}(\Omega)+\operatorname{Re} \sum_{k=1}^{N} \bar{f}\left(\Omega+\frac{\mathrm{i} k \pi}{t_{1}}\right) \exp \left(\frac{\mathrm{i} k \pi t}{t_{1}}\right)\right] \quad 0<t_{1}<2 t
$$

where Re is the real part and i is the imaginary unit. The value of $N$ is chosen sufficiently large and it represents the number in terms of the truncated Fourier series such that

$$
f(t)=\exp (\Omega t) \operatorname{Re}\left[\bar{f}\left(\Omega+\frac{\mathrm{i} N \pi}{t_{1}}\right) \exp \left(\frac{\mathrm{i} N \pi t}{t_{1}}\right)\right] \leqslant \varepsilon_{1}
$$

where $\varepsilon_{1}$ is a prescribed small positive number. Also, the value of $\Omega$ should satisfy the relation $\Omega t \simeq 4.7$ for faster convergence, Tzou (1996).

\section{$\underline{\text { Particular cases }}$}

i) If $\tau_{0}=0$ in equations (5.6), it yields corresponding expressions for the thermoelastic double porous microbeam in context of the coupled theory (CT) of thermoelasticity.

ii) If $b_{1}=\alpha_{3}=\gamma=\alpha_{2}=\gamma_{2}=d \rightarrow 0$ in equations (5.6), we obtain the corresponding expressions for the thermoelastic single porous microbeam (thermoelastic microbeam with voids).

\section{Numerical results and discussion}

Numerical computations have been done for a copper like material microbeam. The material parameters are taken as in Kumar et al. (2015): $\lambda=7.76 \cdot 10^{10} \mathrm{Nm}^{-2}, C^{*}=3.831 \cdot 10^{3} \mathrm{~m}^{2} \mathrm{~s}^{-2} \mathrm{~K}^{-1}$, $\mu=3.86 \cdot 10^{10} \mathrm{Nm}^{-2}, K^{*}=3.86 \cdot 10^{3} \mathrm{Ns}^{-1} \mathrm{~K}^{-1}, T_{0}=298 \mathrm{~K}, \rho=8.954 \cdot 10^{3} \mathrm{Kgm}^{-3}$, $\alpha_{t}=1.78 \cdot 10^{-5} \mathrm{~K}^{-1}, \alpha_{2}=2.4 \cdot 10^{10} \mathrm{Nm}^{-2}, \alpha_{3}=2.5 \cdot 10^{10} \mathrm{Nm}^{-2}, \gamma=1.1 \cdot 10^{-5} \mathrm{~N}, \alpha=1.3 \cdot 10^{-5} \mathrm{~N}$, $\gamma_{2}=0.219 \cdot 10^{5} \mathrm{Nm}^{-2}, \kappa_{1}=0.1456 \cdot 10^{-12} \mathrm{Nm}^{-2} \mathrm{~s}^{2}, b=0.9 \cdot 10^{10} \mathrm{Nm}^{-2}, \alpha_{1}=2.3 \cdot 10^{10} \mathrm{Nm}^{-2}$, $\kappa_{2}=0.1546 \cdot 10^{-12} \mathrm{Nm}^{-2} \mathrm{~s}^{2}, \tau_{0}=0.01 \mathrm{~s}$.

The aspect ratio of the beam is fixed as $L / h=10, a / h=0.5, z=h / 6$. When $h$ is varied, $L$ and $a$ change accordingly with $h$. For the microscale beam, we take the range of the beam length $L=(1-10) \cdot 10^{-6} \mathrm{~m}$. The plots are prepared by using the dimensionless variables for a wide range of the beam length when, unless otherwise stated, $L=4.0, a=h / 2$ and $z=h / 6$.

The software MATLAB has been used to find the values of axial displacement, lateral deflection, volume fraction field and temperature distribution. Variations of these quantities with respect to the axial distance have been shown in Figs. 2-5 to indicate the effects of velocity and time parameters. In Figs. 2 and 3, solid line, small and big dashes lines correspond to the values of velocity $v=1.0,2.0$ and 3.0, respectively with the fixed value of time $t=0.15$, whereas in 
Figs. 4 and 5, solid line, small and big dashes lines correspond to the values of time $t=0.15$, 0.175 and 0.2 , respectively with the fixed value of velocity $v=1.0$.

Effect of the velocity parameter

In Fig. 2a, it is noticed that the value of axial displacement $u$ initially decreases in $0<x<1$, then increases in $1 \leqslant x<2.8$ and again decreases slowly and steadily in the remaining region. It is also found that the magnitude of $u$ decreases with an increase in the value of velocity $v$ near the source application point while the trend gets reversed as moving away from the source. Figure $2 \mathrm{~b}$ depicts that the lateral deflection $w$ decreases in $0<x<1$, increases in $1 \leqslant x \leqslant 2.2$ and then becomes stationary as $x \geqslant 2.2$. The amplitude of variation is higher near the point of application of the source while as moving away from the source, the values become almost stationary for all the values of velocity $v$. Figure 3 a shows that the volume fraction field $\varphi$ initially decreases sharply in $0<x<1$, then increases abruptly in $1 \leqslant x<3.5$ and then decreases slowly and steadily in the remaining region. Also, the magnitude of $\varphi$ decreases as $v$ increases near the source application point while the trend gets reversed away from the source. From Fig. 3b, it is clear that the value of temperature distribution $T$ initially increases in the region $0<x<1$ and decreases monotonically as $x \geqslant 1$. It is also evident that the magnitude of $T$ increases with an increase in velocity $v$.

(a)

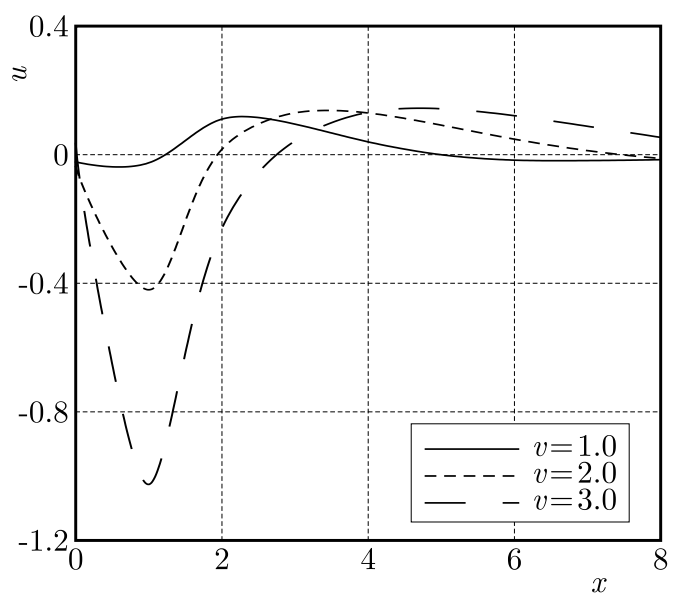

(b)

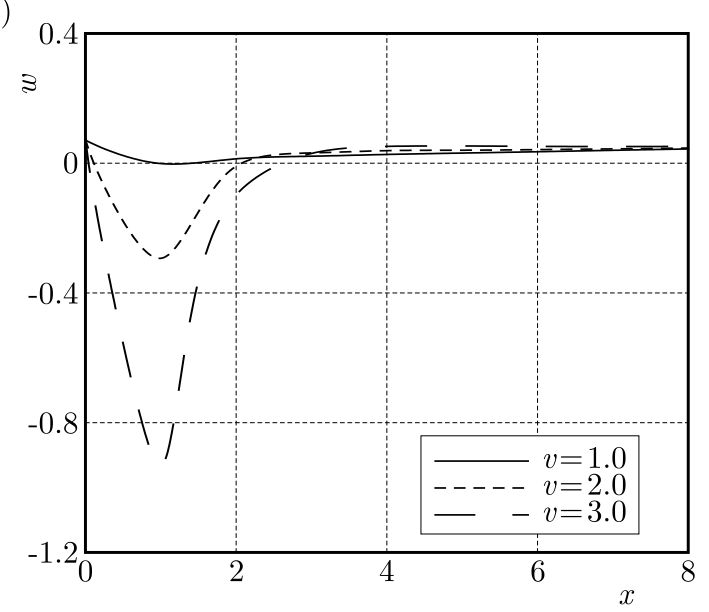

Fig. 2. (a) Axial displacement $u$ and (b) lateral deflection $w$ versus axial distance $x$

(a)

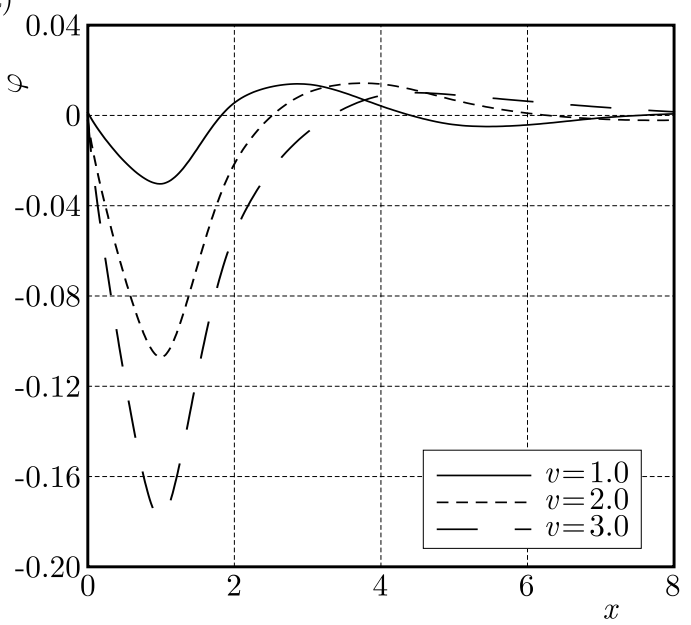

(b)

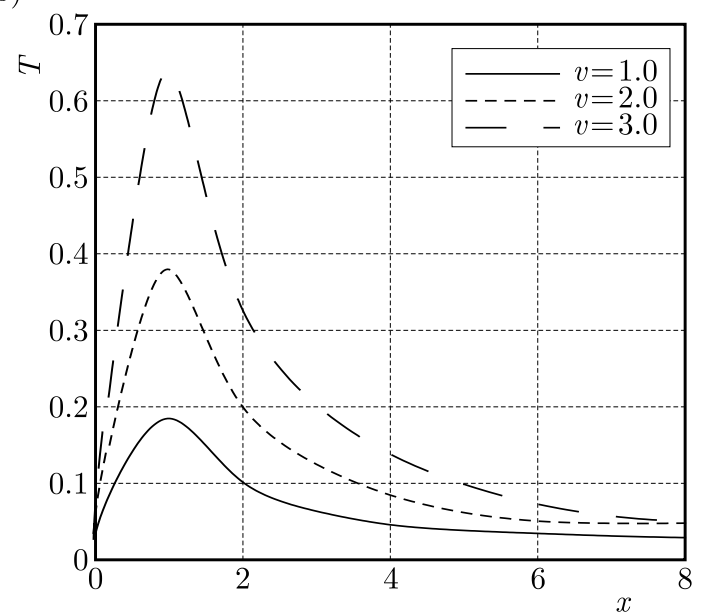

Fig. 3. (a) Volume fraction field $\varphi$ and (a) temperature distribution $T$ versus axial distance $x$ 
Effect of the time parameter

In Fig. 4a, it is noticed that the value of axial displacement $u$ initially decreases in $0<x<1$, then increases in $1 \leqslant x<2.8$ and again decreases in the remaining region. Also, the magnitude of $u$ increases with an increase in the value of time $t$. Figure $4 \mathrm{~b}$ depicts that the lateral deflection $w$ decreases in $0<x<1$ and then increases afterwards as $x \geqslant 1$. It is found that as time $t$ increases, the magnitude of $w$ decreases. Figure 5 a shows that the volume fraction field $\varphi$ is oscillatory in nature. The value of $\varphi$ initially decreases in the range $0<x<1$, then increases in $1 \leqslant x<3.5$ and then decreases in $3.5 \leqslant x<5.3$ and again starts increasing slowly as $x \geqslant 5.3$. The magnitude value of $\varphi$ also increases with an increase in the value of time $t$. From Fig. $5 \mathrm{~b}$, it is clear that the value of temperature distribution $T$ initially increases in the region $0<x<1$ and decreases monotonically as $x \geqslant 1$. It is also found that the magnitude of $T$ decreases with an increase in the value of time $t$.

(a)

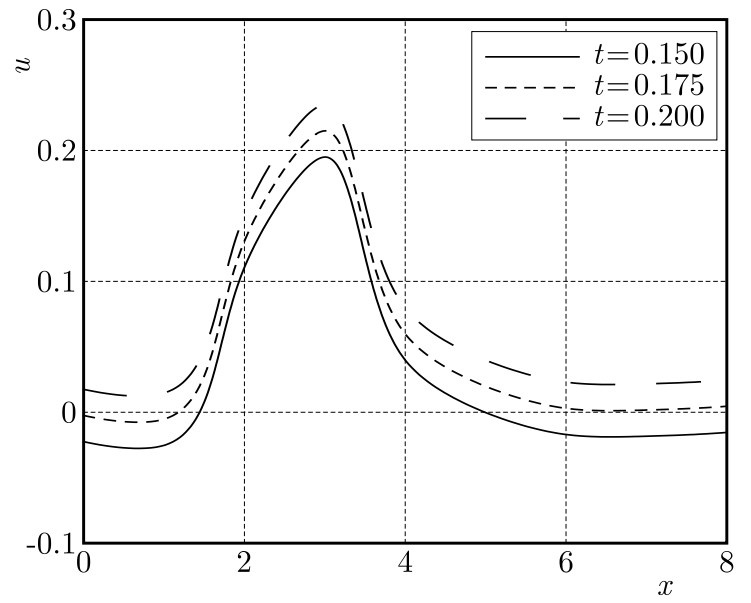

(b)

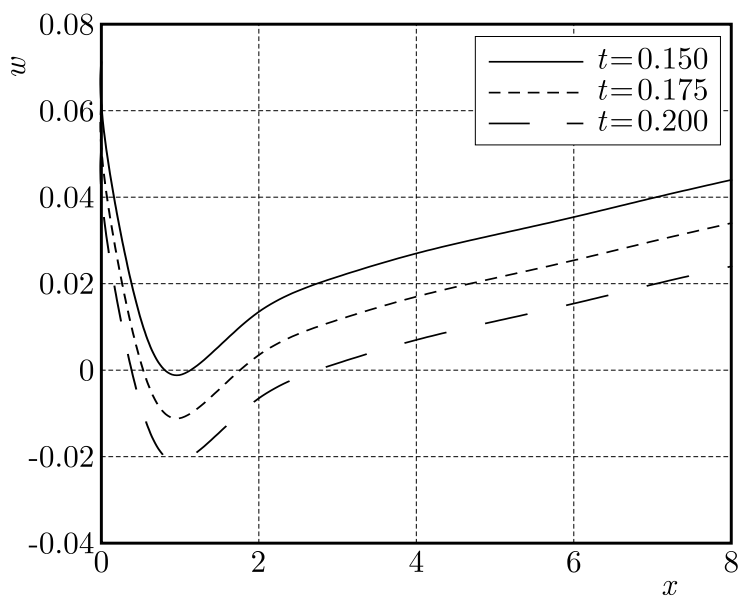

Fig. 4. (a) Axial displacement $u$ and (b) lateral deflection $w$ versus axial distance $x$

(a)

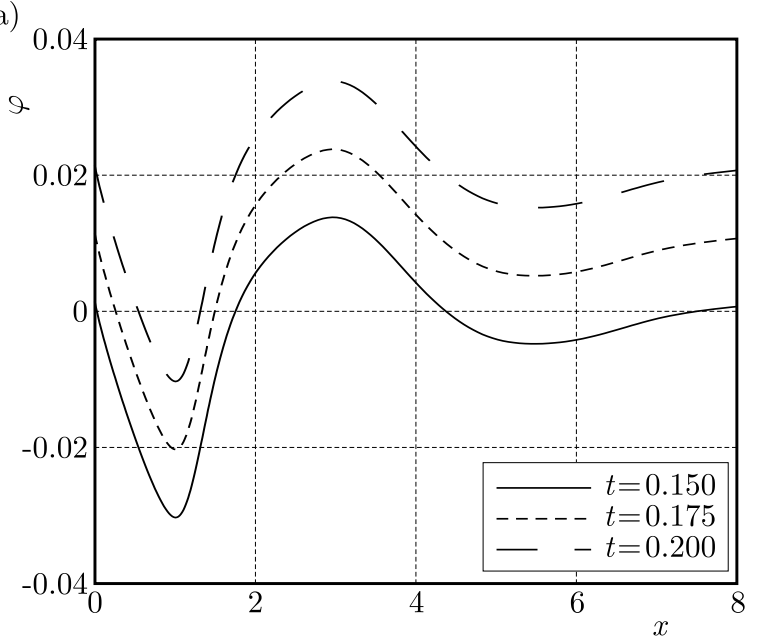

(b)

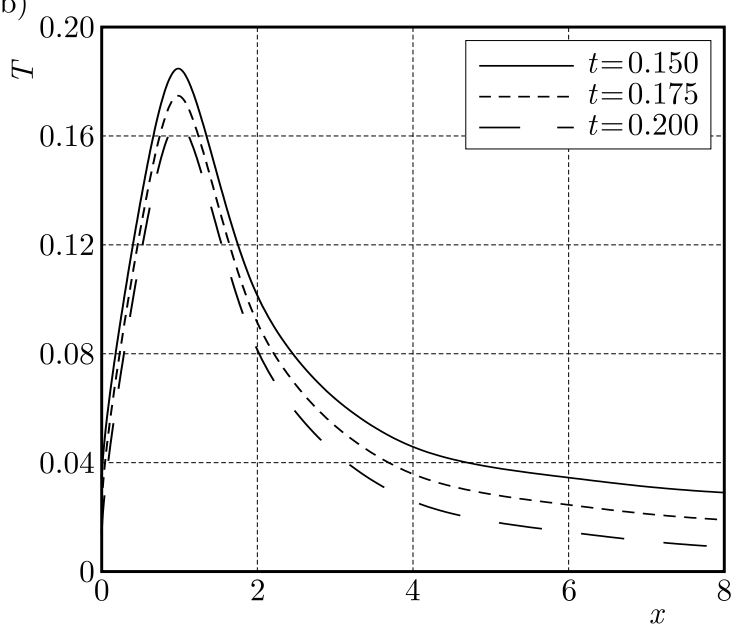

Fig. 5. (a) Volume fraction field $\varphi$ and (b) temperature distribution $T$ versus axial distance $x$ 


\section{Conclusions}

In the present work, forced vibrations of an Euler-Bernoulli thermoelastic double porous microbeam, in context of Lord-Shulman theory of thermoelasticity, subjected to moving load has been investigated. Effects of velocity and time parameters are shown graphically on axial displacement, lateral deflection, volume fraction field and temperature distribution. All field quantities are observed to be very sensitive towards the velocity as well as time parameters.

- It is observed that the amplitude of variation is higher near the application point of the source while the values become almost stationary as moving away from the source due to the effect of velocity. The values of axial displacement $u$ and volume fraction field $\varphi$ decrease with an increase in the velocity $v$ near the application point of the source, whereas an opposite trend of variation is noticed away from the source. The values of lateral deflection $w$ also decrease as the velocity parameter increases near the point of application of the source and becomes stationary as moving away from the source. The magnitude of temperature distribution $T$ gets greater with an increase in the velocity parameter.

- Due to the effect of time parameter, the values of axial displacement $u$ and volume fraction field $\varphi$ increase with an increase in the value of time $t$, whereas an opposite trend and behavior of variation is observed in the case of lateral deflection $w$ and temperature distribution $T$, i.e. the values of $w$ and $T$ decrease as there is an increase in the value of time $t$.

This type of study is useful due to its physical application in many fields of engineering like civil, mechanical, aerospace and industrial sectors. The results obtained in this investigation should prove to be beneficial for researchers working on the theory of thermoelasticity with the double porosity structure. The introduction of the double porous parameter to the thermoelastic medium represents a more realistic model for further studies.

\section{Appendix I}

$$
\begin{aligned}
& a_{26}=s\left(1+\tau_{0} s\right) \quad a_{27}=-s\left(1+\tau_{0} s\right) a_{22} \quad a_{28}=-s\left(1+\tau_{0} s\right) a_{23} \\
& a_{29}=-s\left(1+\tau_{0} s\right) a_{24} \quad a_{30}=-\left[a_{21}+s\left(1+\tau_{0} s\right) a_{25}\right] \\
& n_{1}=-\left(a_{6}+a_{10}+s^{2}\right) \quad n_{2}=-\left(a_{8}+a_{11}\right) \\
& n_{3}=-\left(a_{18}+a_{14}\right) \quad n_{4}=-\left(a_{16}+a_{19}+s^{2}\right) \\
& r_{1}=a_{5} a_{15}-a_{7} a_{13} \quad r_{2}=a_{5}\left(a_{15} a_{30}+n_{4}\right)-a_{13} n_{2}+n_{1} a_{15}-a_{7}\left(a_{13} a_{30}+n_{3}\right) \\
& r_{3}=n_{1}\left(a_{15} a_{30}+n_{4}\right)+a_{5}\left(n_{4} a_{30}-a_{20} a_{29}\right)-a_{7}\left(n_{3} a_{30}-a_{20} a_{28}\right)-n_{2}\left(a_{13} a_{30}+n_{3}\right) \\
& +a_{12}\left(a_{13} a_{29}-a_{15} a_{28}\right) \\
& r_{4}=n_{1}\left(n_{4} a_{30}-a_{20} a_{29}\right)+a_{12}\left(n_{3} a_{29}-n_{4} a_{28}\right)+n_{2}\left(a_{20} a_{28}-n_{3} a_{30}\right) \\
& r_{5}=a_{9} a_{15}-a_{7} a_{17} \quad r_{6}=a_{9}\left(a_{15} a_{30}+n_{4}\right)-a_{7}\left(a_{17} a_{30}-a_{20} a_{27}\right)-n_{2} a_{17}-a_{12} a_{15} a_{27} \\
& r_{7}=a_{9}\left(n_{4} a_{30}-a_{20} a_{29}\right)+a_{12}\left(a_{17} a_{29}-n_{4} a_{27}\right)-n_{2}\left(a_{17} a_{30}-a_{20} a_{27}\right) \\
& r_{8}=a_{9} a_{13}-a_{5} a_{17} \quad r_{9}=a_{9}\left(a_{13} a_{30}+n_{3}\right)-n_{1} a_{17}-a_{5}\left(a_{17} a_{30}-a_{20} a_{27}\right)-a_{12} a_{10} a_{27} \\
& r_{10}=a_{9}\left(n_{3} a_{30}-a_{20} a_{28}\right)-n_{1}\left(a_{30} a_{17}-a_{27} a_{20}\right)+a_{12}\left(a_{17} a_{28}-n_{3} a_{27}\right) \\
& r_{11}=a_{27}\left(a_{5} a_{12}+a_{7} a_{13}\right) \\
& r_{12}=a_{9}\left(a_{13} a_{29}-a_{15} a_{28}\right)+a_{5}\left(n_{4} a_{27}-a_{17} a_{29}\right)+a_{27}\left(n_{1} a_{15}+g_{3} a_{13}\right)-a_{7}\left(a_{17} a_{28}-n_{3} a_{27}\right) \\
& r_{13}=a_{9}\left(n_{3} a_{29}-n_{4} a_{28}\right)-n_{1}\left(a_{17} a_{29}-n_{4} a_{27}\right)-n_{2}\left(a_{17} a_{28}-n_{3} a_{27}\right)
\end{aligned}
$$




$$
\begin{array}{lc}
B_{1}=\frac{r_{2}+a_{2} r_{5}-a_{3} r_{8}-a_{4} r_{11}}{r_{1}} \quad B_{2}=\frac{a_{1} r_{1} s^{2}+a_{2} r_{6}-a_{3} r_{9}-a_{4} r_{12}+r_{3}}{r_{1}} \\
B_{3}=\frac{a_{1} r_{3} s^{2}+a_{2} r_{7}-a_{3} r_{10}-a_{4} r_{13}+r_{4}}{r_{1}} & B_{4}=\frac{a_{1} r_{3} s^{2}}{r_{1}} \quad B_{5}=\frac{a_{1} r_{4} s^{2}}{r_{1}} \\
f_{1}=\frac{1}{v^{7}} F_{0}\left(r_{1} s^{6}+r_{2} s^{4} v^{2}+r_{3} s^{2} v^{4}+r_{4} v^{6}\right) & f_{2}=-\frac{1}{v^{7}} F_{0}\left(r_{5} s^{6}+r_{6} s^{4} v^{2}+r_{7} v^{4} s^{2}\right) \\
f_{3}=\frac{1}{v^{7}} F_{0}\left(r_{8} s^{6}+r_{9} s^{4} v^{2}+r_{10} v^{4} s^{2}\right) & f_{4}=-\frac{1}{v^{7}} F_{0}\left(r_{11} s^{6}+r_{12} s^{4} v^{2}+r_{13} v^{4} s^{2}\right)
\end{array}
$$

\section{Appendix II}

$$
\begin{aligned}
g_{1 i}=-\frac{r_{5} m_{i}^{6}+r_{6} m_{i}^{4}+r_{7} m_{i}^{2}}{r_{1} m_{i}^{6}+r_{2} m_{i}^{4}+r_{3} m_{i}^{2}+r_{4}} & g_{2 i}=\frac{r_{8} m_{i}^{6}+r_{9} m_{i}^{4}+r_{10} m_{i}^{2}}{r_{1} m_{i}^{6}+r_{2} m_{i}^{4}+r_{3} m_{i}^{2}+r_{4}} \\
g_{3 i}=-\frac{r_{11} m_{i}^{6}+r_{12} m_{i}^{4}+r_{13} m_{i}^{2}}{r_{1} m_{i}^{6}+r_{2} m_{i}^{4}+r_{3} m_{i}^{2}+r_{4}} & i=1,2, \ldots, 5
\end{aligned}
$$

\section{References}

1. Barenblatt G.I., Zheltov I.P., Kochina I.N., 1960, Basic concept in the theory of seepage of homogeneous liquids in fissured rocks (strata), Journal of Applied Mathematics and Mechanics, 24, 1286-1303

2. Вiот M.A., 1941, General theory of three-dimensional consolidation, Journal of Applied Physics, 12, 155-164

3. Chang T.P., LiU Y.N., 1996, Dynamic finite element analysis of a nonlinear beam subjected to a moving load, International Journal of Solids and Structures, 33, 12, 1673-1688

4. Cowin S.C., Nunziato J.W., 1983, Linear elastic materials with voids, Journal of Elasticity, 13, 125-147

5. ESEN I., 2015, A new FEM procedure for transverse and longitudinal vibration analysis of thin rectangular plates subjected to a variable velocity moving load along an arbitrary trajectory, Latin American Journal of Solids and Structures, 12, 808-830

6. Honig G., Hirdes U., 1984, A method for the numerical inversion of the Laplace transforms, Journal of Computational and Applied Mathematics, 10, 113-132

7. IEsan D., Quintanilla R., 2014, On a theory of thermoelastic materials with a double porosity structure, Journal of Thermal Stresses, 37, 1017-1036

8. Kaghazian A., Hajnayeb A., Foruzande H., 2017, Free vibration analysis of a piezoelectric nanobeam using nonlocal elasticity theory, Structural Engineering and Mechanics, 61, 5, 617-624

9. Kargarnovin M.H., Ahmadian M.T., Talookolaei R.A.J., 2012, Dynamics of a delaminated Timoshenko beam subjected to a moving oscillatory mass, Mechanics Based Design of Structures and Machines, 40, 2, 218-240

10. KhaliLi N., 2003, Coupling effects in double porosity media with deformable matrix, Geophysical Research Letters, 30, 22, 2153, DOI: 10.1029/2003GL018544

11. Kumar R., 2016, Response of thermoelastic beam due to thermal source in modified couple stress theory, Computational Methods in Science and Technology, 22, 2, 95-101

12. Kumar R., Vohra R., Gorla M.G., 2015, State space approach to boundary value problem for thermoelastic material with double porosity, Applied Mathematics and Computation, 271, 1038-1052 
13. Lord H., Shulman Y., 1967, A generalized dynamical theory of thermoelasticity, Journal of Mechanics and Physics of Solids, 15, 299-309

14. Mehri B., Davar A., Rahmani O., 2009, Dynamic Green function solution of beams under a moving load with different boundary conditions, Scientia Iranica, 16, 3, 273-279

15. Michaltsos G., Sopianopoulo D., Kounadis A.N., 1996, The effect of moving mass and other parameters on the dynamic response of a simply supported beam, Journal of Sound and Vibration, 191, 357-362

16. Nunziato J.W., Cowin S.C., 1979, A nonlinear theory of elastic materials with voids, Archive of Rational Mechanics and Analysis, 72, 175-201

17. Olsson M., 1991, On the fundamental moving load problem, Journal of Sound and Vibration, 145, 2, 299-307

18. RaO G.W., 2000, Linear dynamics of an elastic beam under moving loads, Journal of Vibration and Acoustics, 122, 3, 281-289

19. Sharma J.N., Grover D., 2011, Thermoelastic Vibrations in micro-/nano-scale beam resonators with voids, Journal of Sound and Vibration, 330, 2964-2977

20. Sherief H., SAleH H., 2005, A half space problem in the theory of generalized thermoelastic diffusion, International Journal of Solids and Structures, 42, 4484-4493

21. Sun Y., FAng D, SAKA M , Soh A.K., 2008, Laser-induced vibrations of micro-beams under different boundary conditions, International Journal of Solids and Structures, 45, 1993-2013

22. Tzou D., 1996, Macro-to-Micro Heat transfer, Taylor \& Francis, Washington DC

23. Zenkour A.M., 2017, Thermoelastic response of a microbeam embedded in visco-Pasternak's medium based on GN-III model, Journal of Thermal Stresses, 40, 2, 198-210 